\title{
Pulmonary embolism: yet another cause of hypoxaemic respiratory failure in COVID-19
}

To the Editor:

Patients admitted to hospital for infection due to the novel 2019 coronavirus (SARS-CoV-2) usually show pneumonia and acute hypoxaemic respiratory failure with a wide range of severity, up to acute respiratory distress syndrome (ARDS) [1]. These patients, especially in the most severe cases requiring mechanical ventilation, carry a high thromboembolic risk; therefore, prophylaxis with low molecular weight heparin is recommended by current guidelines [2]. Here, we report a case of a 43-year-old, otherwise healthy man who had never smoked, who was admitted to hospital for acute respiratory failure due to SARS-CoV-2 pneumonia and who subsequently developed acute pulmonary embolism. The patient initially complained of fever and diarrhoea; after a few days, dyspnoea and posterior chest pain developed, and he was eventually admitted. Arterial blood gas (ABG) analysis revealed acute hypoxaemic respiratory failure (oxygen tension $\left(P_{\mathrm{O}_{2}}\right) 59 \mathrm{mmHg}$, carbon dioxide tension $41 \mathrm{mmHg}$ and $\mathrm{pH} 7.47$ while breathing room air); chest radiography demonstrated pale lung shadowing in the left lower lobe. Routine biochemistry showed moderate lymphopenia $\left(770\right.$ per $\left.\mathrm{mm}^{3}\right)$ and elevated C-reactive protein $(\mathrm{CRP})\left(11.81 \mathrm{mg} \cdot \mathrm{dL}^{-1}\right)$ and D-dimer $\left(4.72 \mu \mathrm{g} \cdot \mathrm{mL}^{-1}\right)$ levels; troponin-T was within the normal range in two consecutive samples. ECG was deemed essentially normal by a cardiology consultant. Physical examination revealed reduced respiratory sound in the lower left hemithorax. Heart examination was normal. There was no leg swelling with negative Bauer and Homans signs in both limbs. The patient was treated with hydroxychloroquine $200 \mathrm{mg}$ twice daily, ceftriaxone $2 \mathrm{mg}$ once daily and oxygen until a target saturation (96\%) was reached; a standard prophylaxis for venous thromboembolism (VTE) with enoxaparin $40 \mathrm{mg}$ once daily was immediately started. Despite fever disappearance and improvement of dyspnoea within few days, the patient still complained of left posterior chest pain. Lung ultrasound showed bilateral pleural effusion (more prominent in the left lung), with few B-lines bilaterally. ABG still showed a relative hypoxaemia $\left(P_{\mathrm{O}_{2}}\right.$ between 60 and $62 \mathrm{mmHg}$ while breathing room air) and blood tests showed, despite an improvement in lymphocyte count and in CRP levels, increased D-dimer values (up to $13.13 \mu \mathrm{g} \cdot \mathrm{mL}^{-1}$ ). The patient had no angina, tachycardia or syncope, and haemodynamics had always been normal. Given the persistence of lung effusion, back chest pain and relative hypoxaemia, we suspected a coexisting pulmonary embolism, and a computed tomography pulmonary angiography (CTPA) was performed, which revealed bilateral emboli, more represented in the lower lobar and segmental branches of the right pulmonary arteries, with bilateral pleural effusion (especially in the left lung), without significant parenchymal consolidations (figure 1). Lower limb vein ultrasound showed no signs of deep vein thrombosis and an echocardiogram was normal. Treatment with fondaparinux $7.5 \mathrm{mg}$ once daily was promptly started and over the following days, an improvement in symptoms and ABGs was observed.

Acute infection is a well-known risk factor for VTE due to a strict connection between inflammation and coagulation [3]. In the $2009 \mathrm{H} 1 \mathrm{~N} 1$ influenza pandemic, an increased rate of VTE has been described, especially in the most severe cases with ARDS. Accordingly, an aggressive full anticoagulation with heparin instead of prophylactic doses has been proposed [4]. However, this approach has not been subsequently extensively validated or recommended in ARDS [5]. In 2019 coronavirus disease

@ERSpublications

Pulmonary embolism represents an overlooked cause of worsening respiratory failure in COVID-19. A regular bedside evaluation for atypical features like pleuritic chest pain or pleural effusion could help identify suspected cases for appropriate management. https://bit.ly/3bbBPqZ

Cite this article as: Nieri D, Lenzini G, Canari Venturi B, et al. Pulmonary embolism: yet another cause of hypoxaemic respiratory failure in COVID-19. ERJ Open Res 2020; 6: 00220-2020 [https:// doi.org/10.1183/23120541.00220-2020]. 


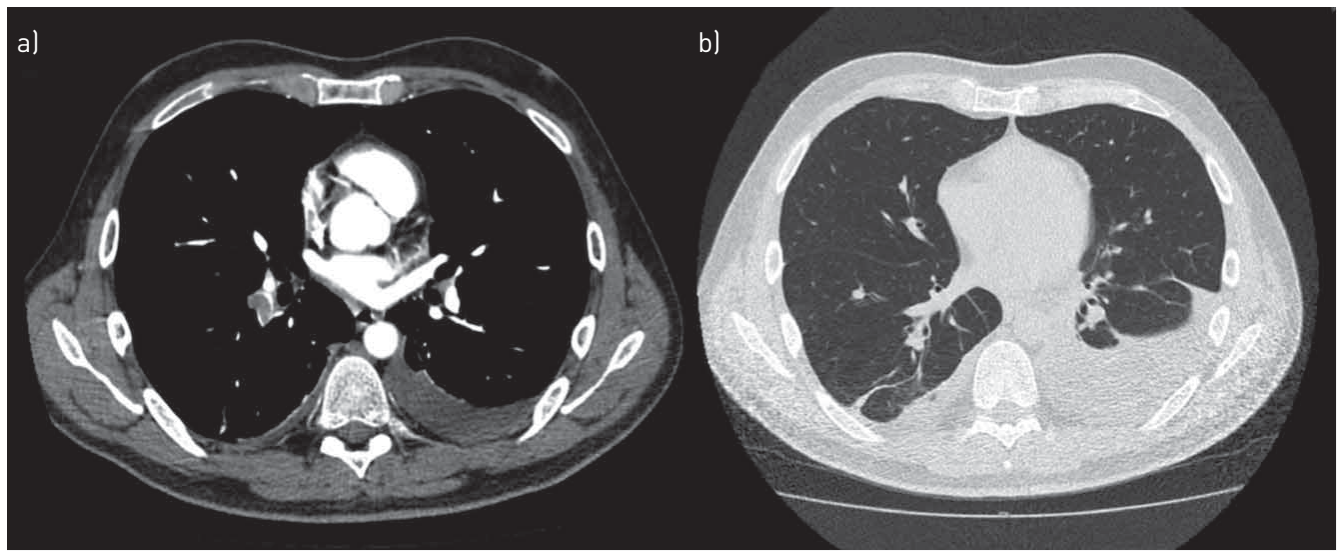

FIGURE 1 a) Computed tomography pulmonary angiography showing acute pulmonary embolism. b) Parenchymal window showing pleural effusion but no ground-glass opacities or consolidation.

(COVID-19) patients, the real rate of VTE is currently not known; however, in a study by Cur et al. [6], 20 (25\%) patients out of 81 admitted to an intensive care unit for severe SARS-CoV-2 pneumonia developed VTE and the authors suggest a possible D-dimer cut off level $\left(1.5 \mu \mathrm{g} \cdot \mathrm{mL}^{-1}\right)$ to identify high-risk subjects, even though the retrospective nature of this observation does not allow firm conclusions to be drawn for routine clinical practice. Moreover, these patients often carry an elevated haemorrhagic risk as well [7], so that prescribing heparin at full anticoagulation dose solely on the basis of D-dimer levels could be potentially dangerous. Raising the clinical suspicion of pulmonary embolism is notoriously challenging; this might be particularly true in a clinical situation, such as COVID-19, in which respiratory failure is a dominant feature, and concerns related to virus containment make it particularly difficult to organise diagnostic tests [8] and even to guarantee appropriate close clinical monitoring. Therefore, it is important to pay particular attention to "atypical" characteristics; pleural effusion, for instance, is a very rare feature in COVID-19 [9], while it is a relatively frequent and often overlooked feature of pulmonary embolism [10] and should therefore prompt attention to possible complications in COVID-19 patients, especially in those without a clear alternative explanation (such as heart failure). Chest pain is a common symptom in pulmonary embolism patients, once again likely not characteristic of COVID-19.

In conclusion, pulmonary embolism must be kept in mind as a possible complication in COVID-19: a regular bedside clinical evaluation to promptly identify atypical features development (including pleuritic chest pain, novel pleural effusion development as assessed by lung ultrasound, sudden onset of tachycardia or right bundle block on ECG) could help to suspect pulmonary embolism, which should ultimately be confirmed by specific diagnostic tests such as CTPA.

Dario Nieri ${ }^{1}$, Giulia Lenzini ${ }^{1}$, Barbara Canari Venturi ${ }^{1}$ and Alessandro Celi ${ }^{2}$

${ }^{1}$ SC Pneumologia, USL Toscana Nordovest, Ospedale San Luca, Lucca, Italy. ${ }^{2}$ Centro Dipartimentale di Biologia Cellulare Cardiorespiratoria; Dipartimento di Patologia Chirurgica, Medica, Molecolare e dell'Area Critica, Università degli Studi di Pisa, Pisa, Italy.

Correspondence: Dario Nieri, SC Pneumologia, Ospedale San Luca, Via Guglielmo Lippi Francesconi 556, 55100 Lucca, Italy. E-mail: darionieri@hotmail.it

Received: 27 April 2020 | Accepted: 30 April 2020

Conflict of interest: D. Nieri has nothing to disclose. G. Lenzini has nothing to disclose. B. Canari Venturi has nothing to disclose. A. Celi reports lecture fees from Boehringer Ingelheim, GSK and Daichi-Sankyo.

\section{References}

1 Yang X, Yu Y, Xu J, et al. Clinical course and outcomes of critically ill patients with SARS-CoV-2 pneumonia in Wuhan, China: a single-centered, retrospective, observational study. Lancet Respir Med 2020; 8: 475-481.

2 Kahn SR, Lim W, Dunn AS, et al. Prevention of VTE in nonsurgical patients: antithrombotic therapy and prevention of thrombosis, 9th ed: American College of Chest Physicians evidence-based clinical practice guidelines. Chest 2012; 141: Suppl., e195S-e226S.

3 Foley JH, Conway EM. Cross talk pathways between coagulation and inflammation. Circ Res 2016; 118: $1392-1408$. 
4 Obi AT, Tignanelli CJ, Jacobs BN, et al. Empirical systemic anticoagulation is associated with decreased venous thromboembolism in critically ill influenza A H1N1 acute respiratory distress syndrome patients. J Vasc Surg Venous Lymphat Disord 2019; 7: 317-324.

5 Rhodes A, Evans LE, Alhazzani W, et al. Surviving sepsis campaign: international guidelines for management of sepsis and septic shock: 2016. Intensive Care Med 2017; 43: 304-377.

6 Cui S, Chen S, Li X, et al. Prevalence of venous thromboembolism in patients with severe novel coronavirus pneumonia. J Thromb Haemost 2020; in press [https://doi.org/10.1111/jth.14830].

7 Wang T, Chen R, Liu C, et al. Attention should be paid to venous thromboembolism prophylaxis in the management of COVID-19. Lancet Haematol 2020; 7: e362-e363.

8 Zuckier LS, Moadel RM, Haramati LB, et al. Diagnostic evaluation of pulmonary embolism during the COVID-19 pandemic. J Nucl Med 2020; 61: 630-631.

9 Shi H, Han X, Jiang N, et al. Radiological findings from 81 patients with COVID-19 pneumonia in Wuhan, China: a descriptive study. Lancet Infect Dis 2020; 20: 425-434.

10 Findik S. Pleural effusion in pulmonary embolism. Curr Opin Pulm Med 2012; 18: 347-354. 